\title{
Development of a Population Pharmacokinetic Model of Vancomycin and its Application in Chinese Geriatric Patients with Pulmonary Infections
}

\author{
Ying Zhou ${ }^{1,2}$. Feifei Gao ${ }^{1,2}$. Chaoyang Chen ${ }^{1}$. Lingyun Ma ${ }^{1}$ Ting Yang ${ }^{1} \cdot$ Xiao Liu ${ }^{1}$ Yaou Liu ${ }^{1}$ Xiaoqing Wang ${ }^{1,2}$. \\ Xia Zhao ${ }^{1}$. Chengli Que ${ }^{3}$. Shuangling $\mathrm{Li}^{4} \cdot \mathrm{JiCheng}^{\mathrm{Li}}{ }^{5} \cdot$ Yimin Cui $^{1,2} \mathbb{D} \cdot \mathrm{Li} \mathrm{Yang}^{5}$
}

Published online: 30 November 2018

(C) The Author(s) 2018

\begin{abstract}
Background The optimal use of vancomycin in the elderly requires information about the drug's pharmacokinetics and the influence of various factors on the drug's disposition. However, because of sampling restrictions, it is often difficult to perform traditional pharmacokinetic studies in elderly patients.

Objective This study was conducted to estimate the population pharmacokinetics of vancomycin in Chinese geriatric patients (age $\geq 65$ years) with pulmonary infections and to explore the clinical application of this information for vancomycin dose individualization.

Methods The steady-state trough concentrations were retrospectively collected from January 2011 to December 2016 and were analyzed using the nonlinear mixed-effect model software. The final model was evaluated using the bootstrap method, goodness-of-fit plots and the normalized prediction distribution error method.

Main Outcome Measure Model parameters and prediction error.

Results A total of 125 steady-state trough concentrations from 70 patients were retrospectively collected. A one-compartment model was established. The final model was depicted as clearance $(\mathrm{CL})[\mathrm{L} / \mathrm{h}]=2.45 \times\left(\mathrm{CL}_{\mathrm{CR}} / 56.28\right) \times 0.542$; volume of distribution $\left(V_{\mathrm{d}}\right)[\mathrm{L}]=154$. The creatinine clearance $\left(\mathrm{CL}_{\mathrm{CR}}\right)$ was identified as the most significant covariate in the final model. The typical values of CL and $V_{\mathrm{d}}$ in the final model were $2.45 \mathrm{~L} / \mathrm{h}$ and $154 \mathrm{~L}$, respectively. Model validation outcomes showed that the final model was stable and had satisfactory prediction performance.

Conclusion A population pharmacokinetic model was established to estimate the pharmacokinetics characteristics of Chinese geriatric patients with pulmonary infections, and this model can be used to develop an initial vancomycin dosing regimen for geriatric patients.
\end{abstract}

Yimin Cui

cui.pharm@pkufh.com

$\triangle$ Li Yang

li.yang@bjmu.edu.cn

1 College of Pharmacy, Peking University Health Science Center, No.38 Xueyuan Road, Haidian District, Beijing, China

2 Department of Pharmacy, Peking University First Hospital, No.6 Dahongluochang Street, Xicheng District, Beijing, China

3 Department of Respiratory Medicine, Peking University First Hospital, No.1 Dahongluochang Street, Xicheng District, Beijing, China

4 Intensive-Care Unit, Peking University First Hospital, No.1 Dahongluochang Street, Xicheng District, Beijing, China

5 Department of Nephrology, Peking University First Hospital, No.1 Dahongluochang Street, Xicheng District, Beijing, China

\section{Key Points}

A population pharmacokinetic model was established to estimate the pharmacokinetics characteristics in Chinese geriatric patients with pulmonary infections.

The model could be used to develop an initial assessment of vancomycin dosing in geriatric patients.

\section{Introduction}

Pulmonary infections are common in hospitalized patients. Vancomycin is the first choice for the treatment of pulmonary infections caused by Gram-positive staphylococcus and 
is considered the last defense against Gram-positive bacterial infections [1]. These infections are usually more severe in geriatric patients than in younger adult patients. Bacteria frequently develop resistance to antibiotics [2]. Drug-resistant bacteria as well as adverse drug reactions are more likely occurrences in the elderly, which means that drug therapy in this population fails or results in death at a higher rate [3].

Vancomycin, a bacteriostatic glycopeptide antibiotic, remains the most frequently used antibiotic in critically ill patients for the treatment of bacterial infections due to methicillin-resistant Staphylococcus aureus. One of the common adverse reactions of vancomycin is acute kidney injury (AKI) $[4,5]$, which has a high incidence and a short incubation period (it may occur in $48 \mathrm{~h}$ ). Presently, the conventional guidance is that therapeutic drug monitoring (TDM) should be carried out within $48 \mathrm{~h}$ after stability, but the warning signs of injury may be delayed past this timeframe. However, if the pharmacokinetics of a patient can be predicted by using a population pharmacokinetic (PopPK) model based on renal function, the dose may be adjusted prior to dispensing the medication so that it is safer and more effective, which may effectively reduce the incidence of vancomycin AKI. Vancomycin-induced acute kidney injury (VI-AKI) occurs more readily in geriatric patients with pulmonary infections, so there is a more urgent need in these patients for an accurate dosage regimen.

The renal function of the elderly gradually decreases with age, which has a significant impact on the pharmacokinetics of vancomycin. A larger volume of distribution and a longer half-life of vancomycin have been observed in elderly patients as compared to younger adult patients [6]. Geriatric patients with pulmonary infections usually have poor nutrition, are associated with hypoalbuminemia, and suffer from internal environmental disorders such as hypokalemia, hyponatremia and metabolic acidosis $[4,6]$. Thus, the elderly and the young are distinguished in terms of their pharmacokinetic characteristics. However, the pharmacokinetics of vancomycin in the elderly population are less known [6].

Currently, there are limited PopPK models for vancomycin studies in geriatric patients. It is necessary to establish a PopPK model and individual treatment plans for elderly patients to improve the safety and effectiveness of vancomycin therapy in geriatric patients.

The objective of the present study was to estimate the PopPK of vancomycin in Chinese geriatric patients (age $\geq 65$ years) with pulmonary infections.

\section{Ethics Approval}

This study was approved by the Clinical Research Ethics Committee of Peking University First Hospital on December 15,2015 . The approval number is 2015 [998].

\section{Methods}

\subsection{Patients and Data Collection}

All patients of Peking University First Hospital between January 2012 and December 2016 who satisfied the screening criteria were included in the study. The clinical data of these patients were retrospectively collected.

The inclusion criteria were as follows: (1) age $\geq 65$ years, (2) patient had received intravenous vancomycin infusion for more than 2 days during hospitalization due to pulmonary infection [hospital-acquired pneumonia (HAP) or community-acquired pneumonia (CAP)], and (3) patients had received TDM more than once.

The exclusion criteria were as follows: (1) patient had multiple organ failure, renal replacement therapy or low volume shock, or (2) patient's clinical data were lacking.

For those patients who qualified for the study, we collected basic information, vancomycin treatment information, TDM information, laboratory test results and information about concomitant drugs. The basic information consisted of sex, age, height and weight; the vancomycin treatment information consisted of dosage, interval, infusion time, and the number of infusions; the TDM information consisted of monitoring time and results; the laboratory test results consisted of serum creatinine, alanine aminotransferase, aspartate aminotransferase, total protein, albumin, total bilirubin, and urea nitrogen; and concomitant drugs consisted of amphotericin B, amikacin, diuretics (furosemide, torsemide, hydrochlorothiazide, spironolactone), and vasoactive drugs (catecholamines, epinephrine, norepinephrine).

\subsection{Blood Sampling and Vancomycin Assays}

The vancomycin dosing regimen consisted of $500 \mathrm{mg}$ (every $6 \mathrm{~h}, 8 \mathrm{~h}, 12 \mathrm{~h}, 24 \mathrm{~h}, 48 \mathrm{~h}$ ) and $1000 \mathrm{mg}$ (every $8 \mathrm{~h}, 12 \mathrm{~h}$ ), for $1.5-2 \mathrm{~h}$ intravenous infusion. The TDM usually occurred $0.5-2 \mathrm{~h}$ before and after the fourth or fifth dose of vancomycin, which were regarded as steady-state concentrations. All the TDM data were collected. All serum concentrations of vancomycin were determined via the chemiluminescence microparticle immunoassay method with the ARCHITECT i1000 system (Abbott Laboratories, Chicago, IL, USA). The coefficient of variation was less than $10 \%$, and the linear range was between 3 and $100 \mathrm{mg} / \mathrm{L}$.

\subsection{PopPK Analysis}

The vancomycin PopPK model was established and simulated with nonlinear mixed effect model software NONMEM ${ }^{\circledR}$ (v.7.3.0; ICON Development Solution, Ellicott City, MD, USA), and the Rstudio environment (v.98.1103, https://www. 
rstudio.com/) was used for statistical analysis and plotting. A first-order conditional estimation method with an inter- and intra-subject variability interaction (FOCE-I) option was used to calculate pharmacokinetic parameters.

The serum concentrations in the study were trough concentrations in steady-state; therefore, we established a one-compartment model that with first-order elimination (ADVAN1 TRANS2) was used as a base model to describe the relationship between serum concentration and time. The inter-individual variability of the model was formulated with an exponential random-effects formula, and the formulae were as follows:

$\mathrm{CL}_{i}=\theta_{\mathrm{CL}} \times \exp (\eta i)$

$V_{\mathrm{di}}=\theta_{V_{\mathrm{d}}} \times \exp (\eta i)$.

$\mathrm{CL}_{\mathrm{i}}$ is the relative clearance of patient $\mathrm{i}, \theta_{\mathrm{CL}}$ the population typical value, and $\eta$ the individual variation. The results show normal distribution with a mean of 0 and a variance of $\omega 2\left(N\left(0, \omega^{2}\right)\right)$.

The residual unexplained variability was fitted with a mixture model. The formula used was as follows:

$C_{i, \mathrm{obs}}=C_{i, \mathrm{pre}} \times\left(1+\varepsilon_{1}\right)+\varepsilon_{2}$,

where $C_{i, \mathrm{obs}}$ is the observed concentration of $i$ th patient, $C_{i, \text { pre }}$ the simulated concentration of the $i$ th patient, $\varepsilon_{1}$, and $\varepsilon_{2}$ residual variabilities that assume a normal distribution in which the mean is 0 and the variance is $\sigma_{1}^{2}\left(N\left(0, \sigma_{1}^{2}\right)\right.$ and $\sigma_{2}^{2}$ $\left(N\left(0, \sigma_{2}^{2}\right)\right), \varepsilon_{1}$ proportional residual variability, and $\varepsilon_{2}$ the additive residual variability.

Table 1 Demographic and pathophysiological characteristics of study patients

\begin{tabular}{|c|c|}
\hline Characteristic & Statistics results \\
\hline Male/female $n$ & $49 / 21$ \\
\hline $\mathrm{AGE} /($ years $)($ mean $\pm \mathrm{SD})$ & $78.3 \pm 6.96$ \\
\hline Height/cm $($ mean \pm SD $)$ & $161 \pm 10$ \\
\hline $\mathrm{WT} / \mathrm{kg}($ mean $\pm \mathrm{SD})$ & $60.7 \pm 10.2$ \\
\hline Daily dose/g/day (mean \pm SD) & $1.55 \pm 0.770$ \\
\hline Serum concentration $/ \mathrm{mg} / \mathrm{L}($ mean $\pm \mathrm{SD})$ & $17 \pm 8.03$ \\
\hline $\mathrm{SCR} / \mu \mathrm{mol} / \mathrm{L}(\operatorname{mean} \pm \mathrm{SD})$ & $90.6 \pm 31$ \\
\hline $\mathrm{CL}_{\mathrm{CR}} / \mathrm{mL} / \min (\operatorname{mean} \pm \mathrm{SD})$ & $56.3 \pm 22.1$ \\
\hline $\mathrm{BUN} / \mathrm{mmol} / \mathrm{L}$ (minimum-maximum) & $10.5(3.18-86)$ \\
\hline $\mathrm{TP} / \mathrm{g} / \mathrm{dL}($ mean $\pm \mathrm{SD})$ & $59.8 \pm 8.92$ \\
\hline $\mathrm{ALB} / \mathrm{g} / \mathrm{dL}($ mean $\pm \mathrm{SD})$ & $29.3 \pm 4.12$ \\
\hline HAP $n(\%)$ & $57(81.4 \%)$ \\
\hline CAP $n(\%)$ & $13(18.6 \%)$ \\
\hline Respiratory failure $n(\%)$ & $46(65.7 \%)$ \\
\hline Hypertension $n(\%)$ & $38(54.3 \%)$ \\
\hline
\end{tabular}

$W T$ weight, $S C R$ serum creatinine, $C L_{C R}$ creatinine clearance rate, $B U N$ blood urea nitrogen, $T P$ total protein, $A L B$ albumin, $H A P$ hospital-acquired pneumonia, $C A P$ community-acquired pneumonia
The covariates examined in this study were sex (SEX), AGE, weight (WT), serum creatinine (SCR), creatinine clearance $\left(\mathrm{CL}_{\mathrm{CR}}\right)$, alanine aminotransferase (ALT) and aspartate aminotransferase (AST), urea nitrogen (BUN), total protein (TP), albumin (ALB) total bilirubin (TBIL), amikacin, amphotericin $\mathrm{B}$, diuretics, and vasoactive drugs. $\mathrm{CL}_{\mathrm{CR}}$ was calculated using the Cockcroft-Gault formula. First, we selected covariates that could significantly improve the fitness of the model. Then, we added covariates into the base model and subsequently observed a change in the minimum value of the objective function (OFV); the covariate significantly enhanced the fitness of the model $\left(\chi^{2}, p<0.05\right)$ once the OFV decreased by more than 3.84 , determining which enhancement could be added into the whole model. The selected covariates were added into the base model in the order of the degree of OFV decrease, starting with the covariate that caused the highest degree.

A backward elimination method was applied to determine the final model, and the covariates were eliminated in the reverse order they were used to build the total quantity model. We observed a change in the minimum value of the OFV. If the OFV increased by more than 6.64 , the covariate significantly improved the fitness of the model $\left(\chi^{2}, p<0.01\right)$, and persisted in the model. The influences of continuous covariates and categorical covariates were validated by formulae as follows:

$\mathrm{CL}_{\mathrm{i}}=\mathrm{TV}(\mathrm{CL}) \times($ covariate/typical value $)$

$\mathrm{CL}_{\mathrm{i}}=\mathrm{TV}(\mathrm{CL})+\theta \times($ covariate \pm typical value $)$

Table 2 Model construction process

\begin{tabular}{|c|c|c|c|c|}
\hline Model ID & OFV & $\Delta \mathrm{OFV}$ & $P$ value & Modeling \\
\hline 1 & 617.755 & - & - & Basic model \\
\hline \multicolumn{5}{|c|}{ Covariates on CL } \\
\hline 2 & 606.197 & -11.558 & $P<0.05$ & $\begin{array}{l}\text { Add } \mathrm{CL}_{\mathrm{CR}} \text { on } \mathrm{CL} \text { in } \\
\text { model } 1\end{array}$ \\
\hline 3 & 608.973 & -8.782 & $P<0.05$ & $\begin{array}{l}\text { Add AGE on CL in } \\
\text { model } 1\end{array}$ \\
\hline 4 & 609.196 & -8.559 & $P<0.05$ & $\begin{array}{l}\text { Add WT on CL in } \\
\text { model } 1\end{array}$ \\
\hline \multicolumn{5}{|c|}{ Forward inclusion } \\
\hline 5 & 601.501 & -4.696 & $P<0.05$ & $\begin{array}{l}\text { Add AGE on CL in } \\
\text { model } 2\end{array}$ \\
\hline 6 & 602.416 & -3.781 & $P>0.05$ & $\begin{array}{l}\text { Add WT on CL in } \\
\text { model } 2\end{array}$ \\
\hline \multicolumn{5}{|c|}{ Backward elimination } \\
\hline 7 & 608.973 & $7.472>6.64$ & $P<0.01$ & $\begin{array}{l}\text { Sub CL } \mathrm{CR}_{\mathrm{CR}} \text { on } \mathrm{CL} \text { in } \\
\text { model } 6\end{array}$ \\
\hline 8 & 606.197 & $4.696<6.64$ & $P>0.01$ & $\begin{array}{l}\text { Sub AGE on CL in } \\
\text { model } 6\end{array}$ \\
\hline & & & & Model 2 as final model \\
\hline
\end{tabular}

$W T$ weight, $C L_{C R}$ creatinine clearance rate, $O F V$ objective function value 

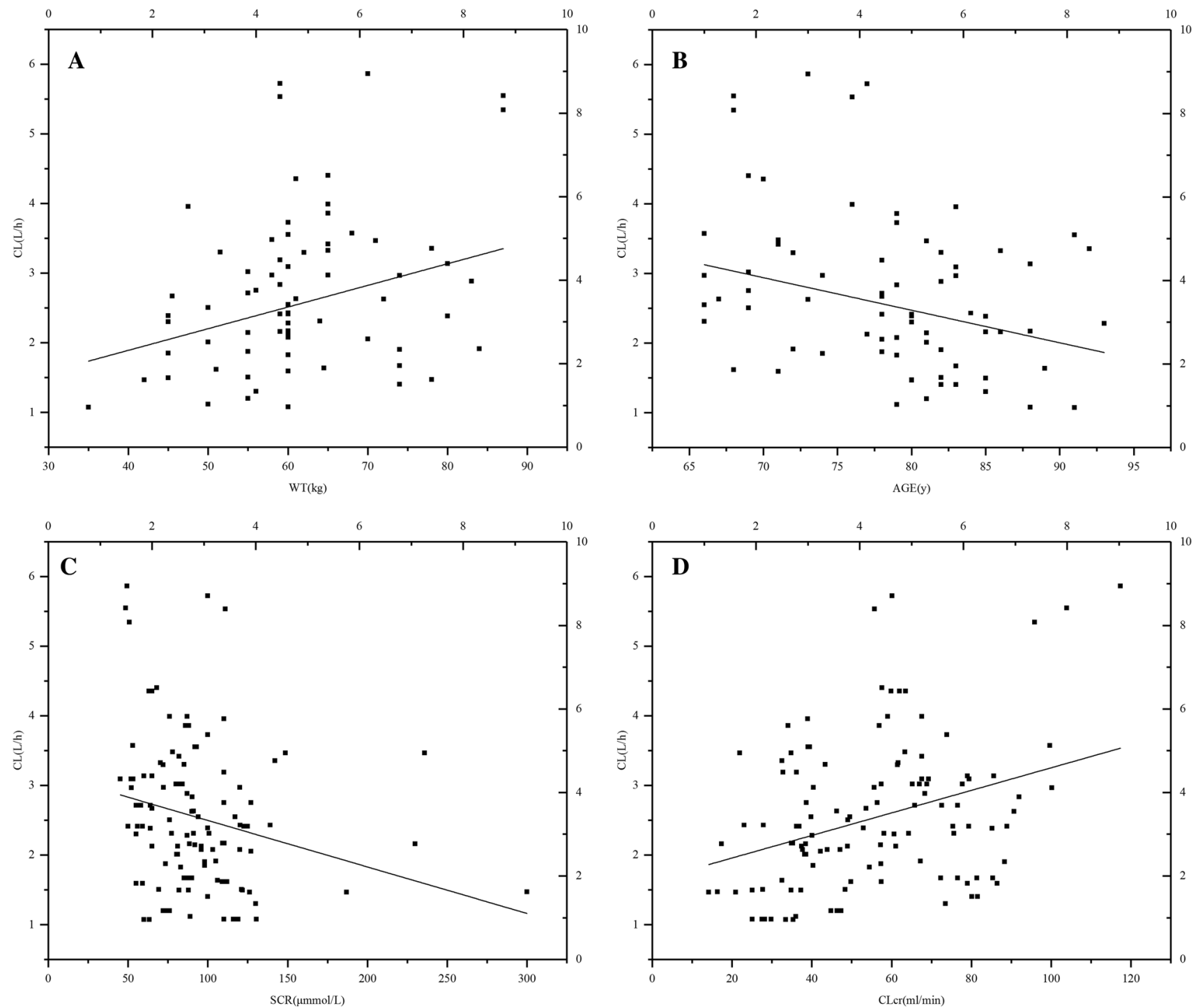

Fig. 1 The correlation analysis between the clearance rate (CL) value of the vancomycin base model and continuous covariates. a Weight (WT) versus $C L$, b AGE versus $C L$, c serum creatinine $(S C R)$ versus $C L, \mathbf{d}$ creatinine clearance rate $\left(C L_{\mathrm{CR}}\right)$ versus $\mathrm{CL}$

$\mathrm{CL}_{\mathrm{i}}=\mathrm{TV}(\mathrm{CL}) \times(\text { covariate } / \text { typical value })^{\theta}$

$\mathrm{CL}_{\mathrm{i}}=\mathrm{TV}(\mathrm{CL}) \mathrm{SEX}=1(1=$ MALE, $2=$ FEMALE $)$

$\mathrm{CL}_{\mathrm{i}}=\mathrm{TV}(\mathrm{CL}) \times \theta \mathrm{SEX}=2$.

\subsection{Model Evaluation}

The internal verification of the final model was carried out by goodness-of-fit plots and statistical methods. Scatter plots were drawn of population-predicted (PRED) and individualpredicted (IPRED) concentrations versus observed concentrations (DV), and TIME versus the conditional weighted residuals (CWRES) and weighted residuals (WRES). A $Y=X$ scatter trend line was added to the IPRED-DV and the
PRED-DV. The closer the slope was to 1 , the better the fit of the final model. The NPDE method was performed with Rstudio (v.0.98.1103, https://www.rstudio.com/). NPDEs were calculated to evaluate the predictive properties of the model. A simulation database was built, and the NPDE parameters were calculated. The mean, variance, skewness and kurtosis of the results were tested with statistical methods to assess whether the distribution approximated the standard normal distribution. We performed statistical tests associated with NPDE that consisted of: (1) a $t$ test to determine whether there was a significant difference between the mean value of NPDE and 0; (2) a Fisher test to determine whether there was a significant difference between NPDE variance and 1; and (3) a Shapiro-Wilks test to determine whether there was a significant difference between the 

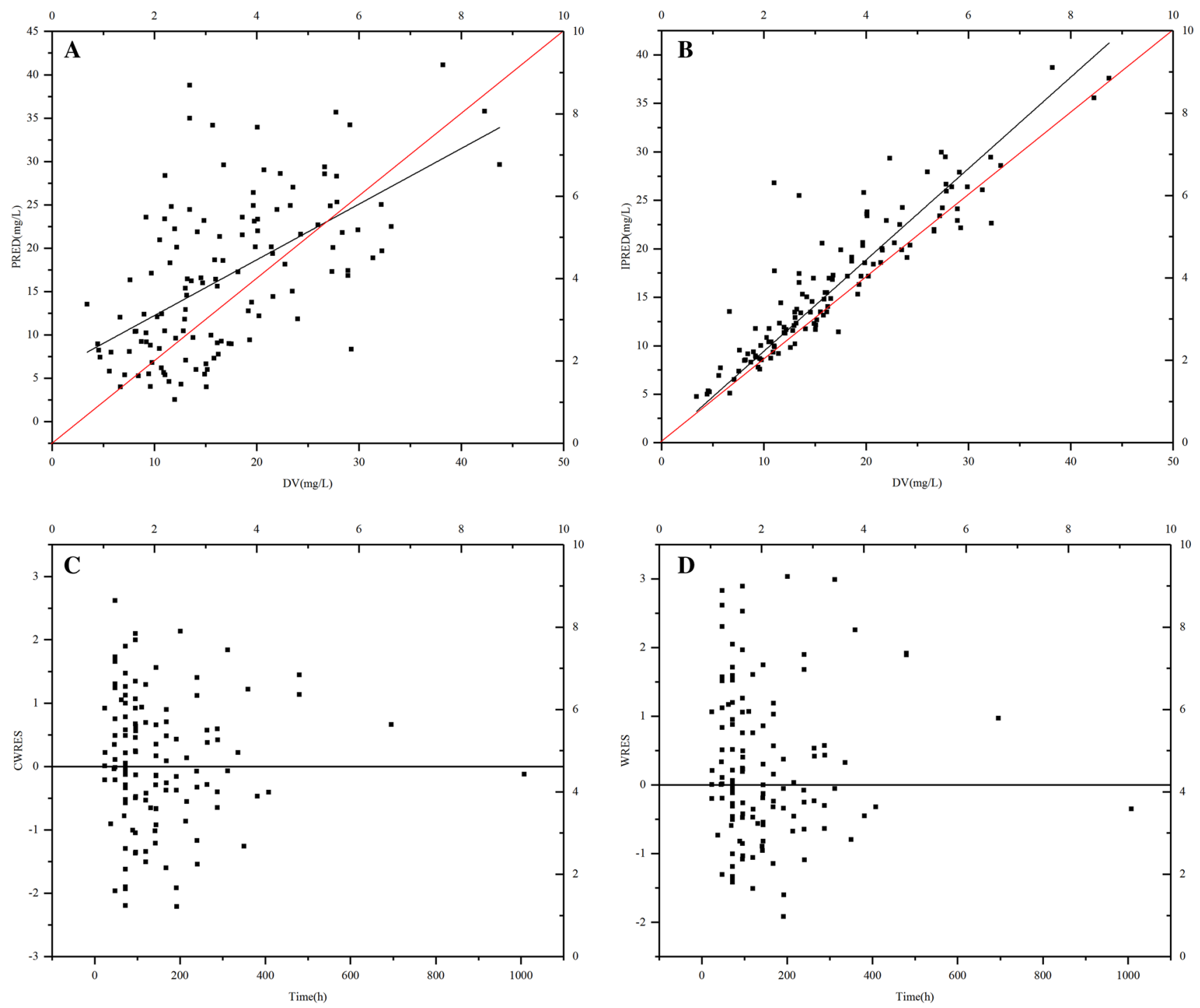

Fig. 2 Diagnostic goodness of fit plots of the final model. a Observed concentration $(D V)$ versus population-predicted concentration $(P R E D)$, b DV versus individual predicted concentration (IPRED),

distribution of NPDE and the normal distribution. Histograms of NPDEs, plots of NPDE versus TIME and NPDE versus predicted concentrations were generated and used as additional simulation-based diagnostics. A total of 1000 bootstrap datasets was generated to test the statistical verification, reliability and stability of the PopPK model. The observed dataset was resampled with replacement data to generate a new dataset with the same size and population characteristics as the original dataset. The robust rate, point estimates and $95 \%$ confidence interval of the model were evaluated. If the results satisfied the conditions of success ratio of 1000 bootstraps $>90 \%$ and each parameter $<95 \%$ confidence interval, the model was considered more stable.

c conditional weighted residuals (CWRES) versus time, d weighted residuals (WRES) versus time

\subsection{Simulation}

We used the simulation module of the NONMEM software to simulate the concentration of vancomycin 1000 times.

\section{Results}

The development of the model was based on a database of 125 observations from 70 . There were 49 males and 21 females in the study. The mean age of patients was 78.3 $( \pm 6.96)$ years. A total of 57 patients $(81.4 \%)$ had HAP while 13 patients $(18.6 \%)$ had CAP. The number of concentration 
data points derived from each patient ranged from 1 to 5 (mean $=1.79$ ). The basic information about the patients is shown in Table 1 . Normally distributed continuous variables were expressed as the mean \pm standard deviation, and nonnormally distributed continuous variables were presented as the median (interquartile range).

The results showed that $\mathrm{CL}_{\mathrm{CR}}$, WT and AGE significantly improved the initial model. Consequently, $\mathrm{CL}_{\mathrm{CR}}$, WT, and AGE were added to build out the whole model. However, when we added AGE to the model, the OFV was $<6.64$, so AGE was not used in the final model.The final model is shown in Eqs. (9) and (10). The modeling process is shown in Table 2. When the influences of continuous covariates were validated by Eq. (3), the OFV was satisfied better.

$\mathrm{CL}(\mathrm{L} / \mathrm{h})=2.45 \times\left(\mathrm{CL}_{\mathrm{CR}} / 56.28\right)^{0.542}$

$V_{d}(\mathrm{~L})=154$.

We drew a scatter plot of continuous covariates and CL fitted by a basic model, and a linear trend line was added to the scatter plot. The results are shown in Fig. 1. SCR and AGE were noted to be inversely related to CL. $\mathrm{CL}_{\mathrm{CR}}$ and WT were noted to be positively related to CL.

We drew scatter plots of PRED and the IPRED versus the DV. We also drew scatter plots of TIME versus WRES or

A

Q.Q plot versus $\mathrm{N}(0,1)$ for $\mathrm{npde}$

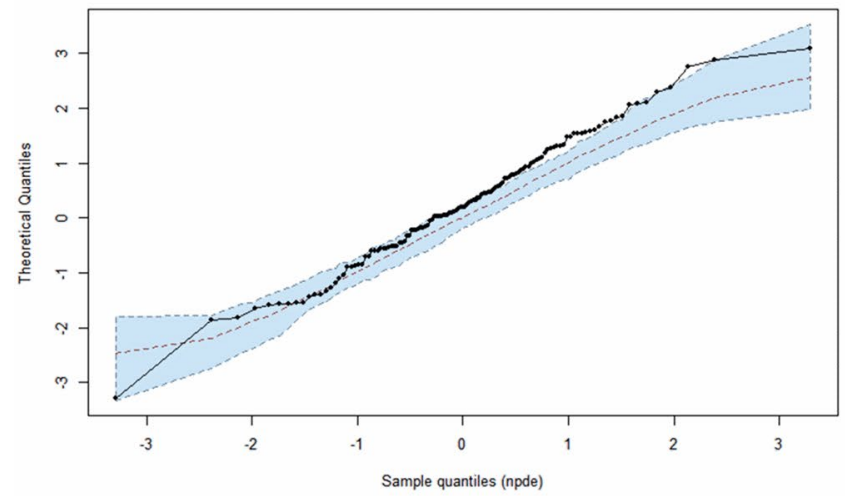

C

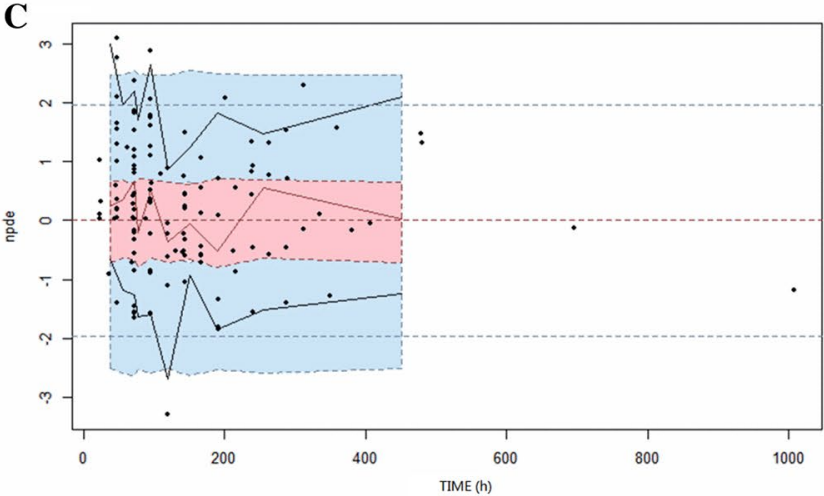

Table 3 Comparison of the base and final models

\begin{tabular}{llllll}
\hline Parameter & \multicolumn{2}{l}{ Final model } & & \multicolumn{2}{l}{ Base model } \\
\cline { 2 - 3 } \cline { 5 - 6 } & Value & RSE\% & & Value & RSE\% \\
\hline$\theta_{1 \mathrm{CL}}(\mathrm{L} / \mathrm{h})$ & 2.45 & $6.9 \%$ & & 2.47 & $7.94 \%$ \\
$\theta_{2 V \mathrm{~d}}(\mathrm{~L})$ & 154 & $9.2 \%$ & & 142 & $17.0 \%$ \\
$\theta_{3 \mathrm{CLCR}}(\mathrm{mL} / \mathrm{min})$ & 0.542 & $35.1 \%$ & - & - \\
$\quad$ on CL & & & & \\
$\omega_{\mathrm{CL}}$ & 0.174 & $21.2 \%$ & 0.235 & $40.6 \%$ \\
$\omega_{V}$ & 0.339 & $37.8 \%$ & 0.213 & $46.8 \%$ \\
$\sigma_{1}$ & 0.0657 & $34.2 \%$ & 0.0722 & $48.8 \%$ \\
$\sigma_{2}$ & 0 FIX & - & 0 FIX & - \\
\hline
\end{tabular}

$\theta_{I C L}$ population parameter of $\mathrm{CL}, \theta_{2 V d}$ population parameter of $V_{\mathrm{d}}$, $\theta_{3 C L C R}$ population parameter of $\mathrm{CL}_{\mathrm{CR}}, \omega_{C L}$ intra-individual variation of $\mathrm{CL}, \sigma_{l}$ proportional residual variation, $\sigma_{2}$ additive residual variation, $C L_{C R}$ creatinine clearance rate

CWRES. The results show that the value of WRES ranges from -3 to 3 , and the value of CWRES ranges from -3 to 3 (see Fig. 2; Table 3).

An internal validation of the NPDE results showed that the mean and variance of the final model are 0.248 and 1.28 , respectively; the values of the $t$ test, Fisher test and Shapiro-Wilks test are $0.0158(p>0.001), 0.0388(p>0.01)$ and

B

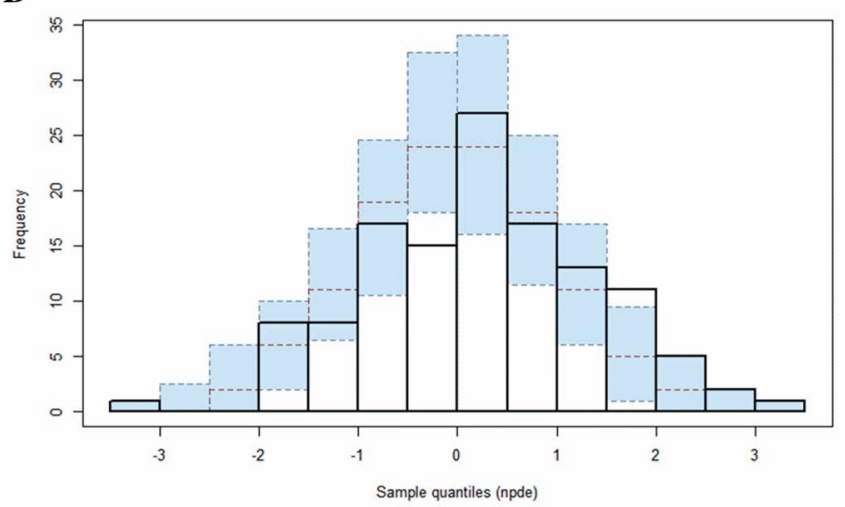

D

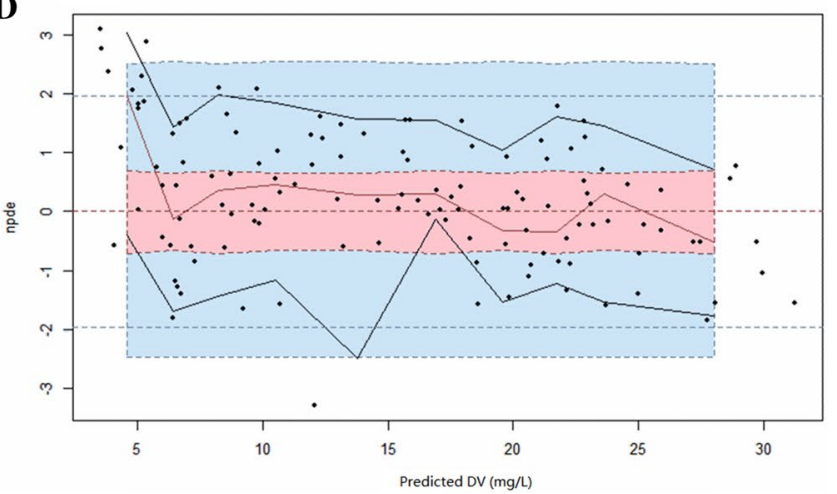

Fig. 3 Normalized predictive distribution error (NPDE) of the population pharmacokinetics final model for vancomycin. a Quantile-quantile plot of NPDE versus normalized distribution, $\mathbf{b}$ the distribution chart of predictive distribution error, $\mathbf{c}$ NPDE versus time, $\mathbf{d}$ NPDE versus PRED 
$0.861(p>0.05)$, respectively. The numerical results show that the distribution of the final model was close to normal distribution, and the model fit well (see Fig. 3). The $y=x$ chart, histogram and random scatter plot showed a similar trend with a normal distribution. The results indicate a good fit, and the 1000 bootstrap statistical verification showed that the model robust rate is $92.1 \%$, the parameter values are all within the $95 \%$ confidence interval, and the $95 \%$ confidence interval estimate did not consist of 0 , indicating that the model was stable. The results are shown in Table 4.

The simulation results show that the initial drug regimen should be established according to the $\mathrm{CL}_{\mathrm{CR}}$ of patients. The results are shown in Table 5. For patients whose $\mathrm{CL}_{\mathrm{CR}}$ is $>50 \mathrm{~mL} / \mathrm{min}$, the initial vancomycin dosing regimen can be the full dose of $1000 \mathrm{mg}$ administered every $8 \mathrm{~h}(18.41 \pm 8.95 \mathrm{mg} / \mathrm{L})$ or q $12 \mathrm{~h}(15.10 \pm 7.02 \mathrm{mg} / \mathrm{L})$. The concentration, however, may exceed $20 \mathrm{mg} / \mathrm{L}$ if the $\mathrm{CL}_{\mathrm{CR}}$ of the patient is not stable at a dosing regimen of $1000 \mathrm{mg}$, every $8 \mathrm{~h}$. For patients whose $\mathrm{CL}_{\mathrm{CR}}$ is $\leq 50 \mathrm{~mL} /$ $\mathrm{min}$, the initial dosing regimen can be modified to $1000 \mathrm{mg}$, q $12 \mathrm{~h}(17.63 \pm 8.44 \mathrm{mg} / \mathrm{L})$ since q $8 \mathrm{~h}$ may lead to a high concentration.

\section{Discussion}

Our study aims to estimate the PopPK model of vancomycin in Chinese geriatric patients (age $\geq 65$ years) with pulmonary infections because there is no suitable PopPK model for this population. After retrospectively collecting 125 observations from 70 patients of Peking University First Hospital, we built the following PopPK model: $\mathrm{CL}(\mathrm{L} / \mathrm{h})=2.45 \times(\mathrm{C}$ $\left.\mathrm{L}_{\mathrm{CR}} / 56.28\right)^{0.542},(9) V_{\mathrm{d}}(\mathrm{L})=154(10)$.

We performed an internal validation and simulation to assess the final model. The distribution of the final model was close to normal distribution, and the model had good fit.

Our study found that $\mathrm{CL}_{\mathrm{CR}}$ is the only covariate that affects the clearance parameter in geriatric patients with pulmonary infections. Overall, $90 \%$ of vancomycin is excreted though the kidney. Renal function is undoubtedly one of the important factors that affects the pharmacokinetics of vancomycin, and the $\mathrm{CL}_{\mathrm{CR}}$ is a substantial factor related to age, weight and creatinine. Additionally, many studies of PopPK models of vancomycin in adults have demonstrated that $\mathrm{CL}_{\mathrm{CR}}$ is one of the most important covariates that affect vancomycin pharmacokinetics. In these studies, the typical value of CL in the final model was $2.45 \mathrm{~L} / \mathrm{h}$. We placed the average values of CL from these studies into our final model
Table 4 Bootstrap results for the final model; 1000 iterations; success rate $92.1 \%$

\begin{tabular}{lllll}
\hline Parameter & $\begin{array}{l}\text { NONMEM } \\
\text { parameter }\end{array}$ & Bootstrap 95\% CI & Bootstrap median & Bootstrap 95\% CI \\
\hline$\theta_{1 \mathrm{CL}}(\mathrm{L} / \mathrm{h})$ & 2.45 & $(2.02,2.88)$ & 2.43 & $(2.09,2.81)$ \\
$\theta_{2 V \mathrm{~d}}(\mathrm{~L})$ & 154 & $(110,197)$ & 154 & $(117,191)$ \\
$\theta_{3 \mathrm{CLCR}}(\mathrm{mL} / \mathrm{min})$ & 0.542 & $(0.141,0.942)$ & 0.538 & $(0.206,0.878)$ \\
$\quad$ on CL & & & & \\
$\omega_{\mathrm{CL}}$ & 0.174 & $(0.076,0.272)$ & 0.162 & $(0.092,0.256)$ \\
$\omega_{V}$ & 0.339 & $(0.079,0.598)$ & 0.289 & $(0.121,0.557)$ \\
$\sigma_{1}$ & 0.0657 & $(0.0177,0.114)$ & 0.0691 & $(0.0254,0.106)$ \\
$\sigma_{2}$ & $0 \mathrm{FIX}$ & - & 0 FIX & - \\
\hline
\end{tabular}

$C L_{C R}$ creatinine clearance rate, $C L$ clearance, $V_{d}$ volume of distribution

\begin{tabular}{lccc}
\hline Dosage regimen & \multicolumn{2}{l}{ Concentration $(\mathrm{mg} / \mathrm{L})$} & \\
\cline { 2 - 4 } & $\begin{array}{l}\text { Total (average plasma } \\
\text { concentration) }\end{array}$ & $\mathrm{CL}_{\mathrm{CR}}>50 \mathrm{~mL} / \mathrm{min}$ & $\mathrm{CL}_{\mathrm{CR}}<50 \mathrm{~mL} / \mathrm{min}$ \\
& $19.26 \pm 9.50$ & $18.41 \pm 8.95$ & $20.72 \pm 9.53$ \\
$1000 \mathrm{mg}$, q 8 h & $16.02 \pm 7.51$ & $15.10 \pm 7.02$ & $17.63 \pm 8.44$ \\
$1000 \mathrm{mg}$, q 12 h & $10.29 \pm 4.69$ & $9.40 \pm 4.38$ & $11.96 \pm 5.30$ \\
$1000 \mathrm{mg}$, q 24 h & $11.22 \pm 5.85$ & $10.83 \pm 4.57$ & $11.88 \pm 4.36$ \\
$500 \mathrm{mg}$, q 6 h & $9.82 \pm 4.97$ & $9.38 \pm 3.65$ & $10.57 \pm 3.57$ \\
$500 \mathrm{mg}$, q 8 h & $7.98 \pm 3.94$ & $7.52 \pm 2.68$ & $8.79 \pm 3.43$ \\
$500 \mathrm{mg}$, q 12 h & $5.06 \pm 2.53$ & $4.62 \pm 1.35$ & $5.89 \pm 2.90$ \\
$500 \mathrm{mg}$, q 24 h & & &
\end{tabular}

$C L_{C R}$ creatinine clearance rate, $q \times h$ every $\times$ hours
Table 5 Simulation data $($ simulation times $=1000)$ 


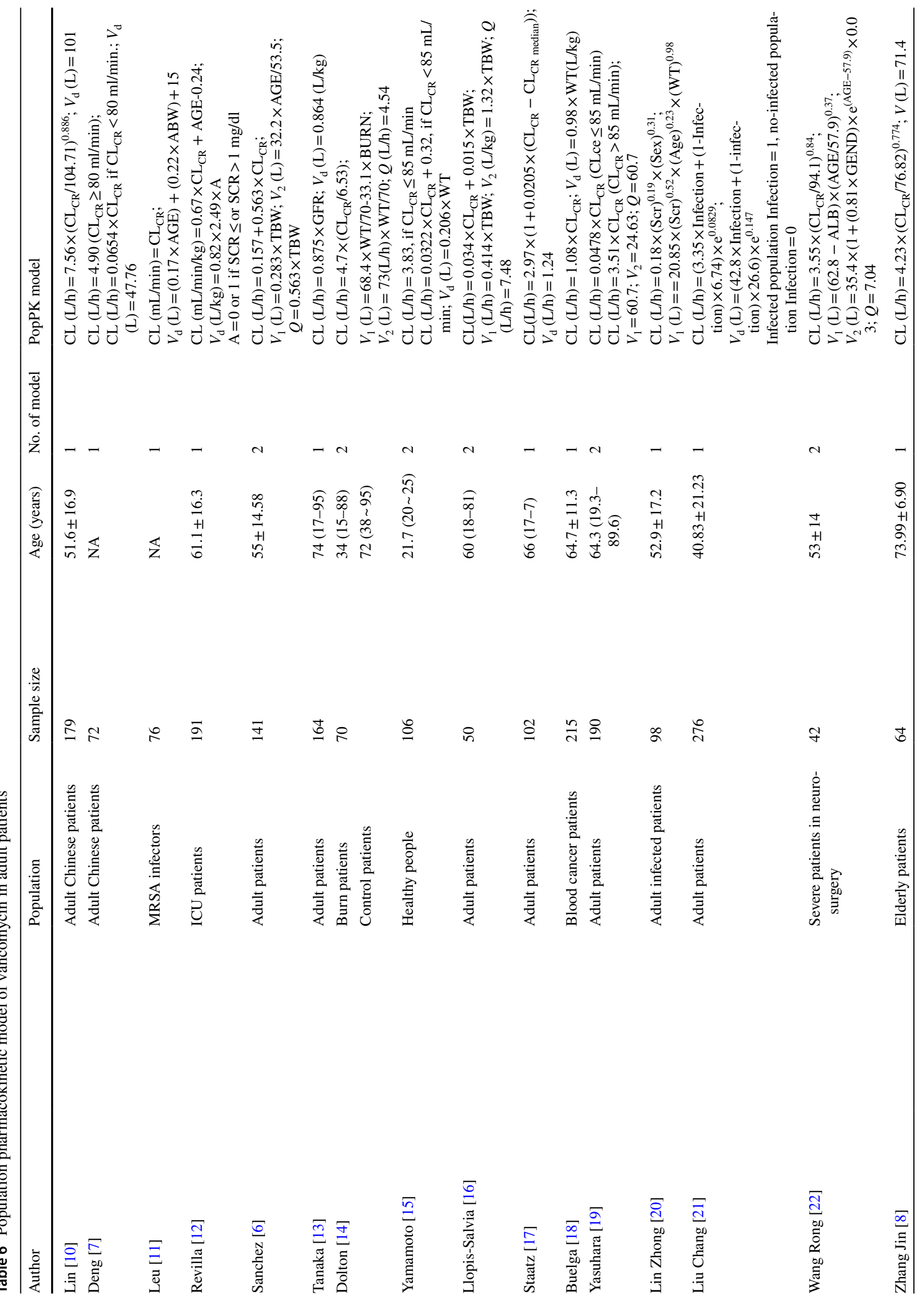




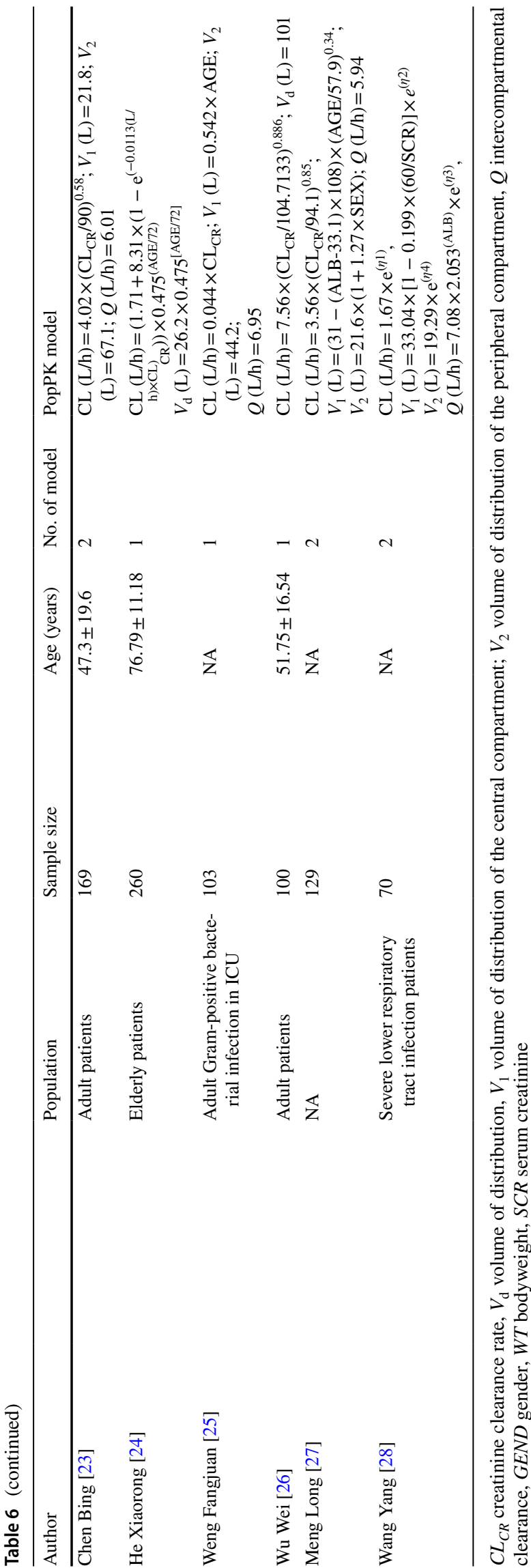

and obtained the typical values of $3.58 \mathrm{~L} / \mathrm{h}$ and $2.95 \mathrm{~L} / \mathrm{h}[8$, 9]. In the study that found a typical value of $3.58 \mathrm{~L} / \mathrm{h}$, the subjects were adult patients. However, research has shown that the $C L$ values of geriatric patients are smaller than those of adult patients [3]. In the study that found a typical value of $2.95 \mathrm{~L} / \mathrm{h}$, the subjects were geriatric patients and the typical values were similar. The models from all the studies we reference are shown in Table 6.

The simulation results show that the initial dosing regimen should be made according to the $\mathrm{CL}_{\mathrm{CR}}$ of patients. For patients whose $\mathrm{CL}_{\mathrm{CR}}$ is $>50 \mathrm{~mL} / \mathrm{min}$, the initial regimen can be $1000 \mathrm{mg}$, q $8 \mathrm{~h}(18.41 \pm 8.95 \mathrm{mg} / \mathrm{L})$ or q $12 \mathrm{~h}$ $(15.10 \pm 7.02 \mathrm{mg} / \mathrm{L})$. However, the concentration may exceed $20 \mathrm{mg} / \mathrm{L}$ with dosing of $1000 \mathrm{mg}$, q $8 \mathrm{~h}$ if the $\mathrm{CL}_{\mathrm{CR}}$ of the patient is not stable. For patients whose $\mathrm{CL}_{\mathrm{CR}}$ is $\leq 50 \mathrm{~mL} / \mathrm{min}$, the initial dosing regimen can be $1000 \mathrm{mg}$, q $12 \mathrm{~h}(17.63 \pm 8.44 \mathrm{mg} / \mathrm{L})$ since $\mathrm{q} 8 \mathrm{~h}$ may lead to a high concentration.

There are some limitations to our study. The concentration values reflect only steady-state trough concentrations since the concentration data were obtained from routine TDM. Furthermore, our study was a retrospective study, which may affect the accuracy of some of the data and influence the final model. Further research, including a prospective study, should be carried out to build a more precise model.

\section{Conclusion}

A one-compartment PopPK model of vancomycin in Chinese geriatric patients with pulmonary infections was established. $\mathrm{CL}_{\mathrm{CR}}$ was found to be the only covariate that affected vancomycin pharmacokinetic parameters. The predictive performance of the PopPK model in geriatric patients differed significantly from that of the PopPK model in adult patients.

\section{Compliance with Ethical Standards}

Funding This study was funded by the Interdisciplinary Clinical Research Project of Peking University First Hospital, under the funding number 2017CR25.

Conflict of interest Zhou Ying, Gao Feifei, Chen Chaoyang, Ma Lingyun, Yang Ting, Liu Xiao, Liu Yaou, Wang Xiaoqing, Zhao, Li Shuangling, Lv JiCheng, Cui Yimin and Yang Li have no conflicts of interest.

Ethics Approval All procedures in this study were conducted in accordance with the 1964 Helsinki Declaration (and its amendments), and the guidelines of the Ethics Committee or institutional review board that approved the study.

Informed Consent Written informed consent was obtained from all patients. 
Open Access This article is distributed under the terms of the Creative Commons Attribution-NonCommercial 4.0 International License (http://creativecommons.org/licenses/by-nc/4.0/), which permits any noncommercial use, distribution, and reproduction in any medium, provided you give appropriate credit to the original author(s) and the source, provide a link to the Creative Commons license, and indicate if changes were made.

\section{References}

1. Michael J, Ben M, John C, et al. Vancomycin therapeutic guidelines: a summary of consensus recommendations from the Infectious Diseases Society of America, the American Society of Health-System Pharmacists, and the Society of Infectious Diseases Pharmacists. Vancomycin Ther Guidelines. 2009;49(01):325-7.

2. Levine DP. Vancomycin: a history. Clin Infect Dis. 2006;42(01):S5-12.

3. Avent ML, Vaska VL, Rogers BA, et al. Vancomycin therapeutics and monitoring: a contemporary approach. Intern Med J. 2013;43(02):110-9.

4. Sheng XY, Gao FF, Ma LY, et al. Investigation of vancomycin used in geriatric patients. Chin J Clin Pharmacol. 2015;31(24):2451-3.

5. Yuzo O, Petrus Z, Dantas MC, et al. Currently used dosage regimens of vancomycin fail to achieve therapeutic levels in approximately $40 \%$ of intensive care unit patients. Rev Bras Ter Intensiva. 2016;28(04):380-6.

6. Sánchez JL, Dominguez AR, Lane JR, et al. Population pharmacokinetics of vancomycin in adult and geriatric patients: comparison of eleven approaches. Int J Clin Pharmacol Ther. 2010;48(8):525-33.

7. Deng C, Zhou T, Cheng D, et al. Initial dosage regimens of vancomycin for Chinese adult patients based on population pharmacokinetic analysis. Int J Clin Pharmacol Ther. 2013;51(05):407-15.

8. Zhang J. Population pharmacokinetics analysis of vancomycin in elderly patients. Fujian: Fujian Medical University; 2014.

9. Pan KM, Ma LY, Xiang Q, et al. Current situation survey and risk factors of vancomycin-associated acute kidney injury in older patients. Chin J New Drugs. 2017;26(15):1848-56.

10. Lin WW, Wu W, Jiao Z, et al. Population pharmacokinetics of vancomycin in adult Chinese patients with post-craniotomy meningitis and its application in individualised dosage regimens. Eur J Clin Pharmacol. 2015;72(01):29-37.

11. Leu WJ, Liu YC, Wang HW, et al. Evaluation of a vancomycin dosing nomogram in achieving high target trough concentrations in Taiwanese patients. Int J Infect Dis. 2012;16(11):e804-10.

12. Revilla N, Martín-Suárez A, Pérez MP, et al. Vancomycin dosing assessment in intensive care unit patients based on a population pharmacokinetic/pharmacodynamic simulation. Br J Clin Pharmacol. 2010;70(02):201-12.
13. Tanaka A, Aiba T, Otsuka T, et al. Population pharmacokinetic analysis of vancomycin using serum cystatin $\mathrm{C}$ as a marker of renal function. Antimicrob Agents Chemother. 2010;54(02):778-82.

14. Dolton $\mathrm{M}, \mathrm{Xu} \mathrm{H}$, Cheong E, et al. Vancomycin pharmacokinetics in patients with severe burn injuries. Burns. 2010;36(04):469-76.

15. Yamamoto M, Kuzuya T, Baba H, et al. Population pharmacokinetic analysis of vancomycin in patients with gram-positive infections and the influence of infectious disease type. J Clin Pharm Ther. 2009;34(04):473-83.

16. Llopis-Salvia P, Jimenez-Torres NV. Population pharmacokinetic parameters of vancomycin in critically ill patients. J Clin Pharm Ther. 2006;31(05):447-54.

17. Staatz CE, Byrne C, Thomson AH. Population pharmacokinetic modelling of gentamicin and vancomycin in patients with unstable renal function following cardiothoracic surgery. Br J Clin Pharmacol. 2006;61(02):164-76.

18. Buelga DS, Herrera EV, Dominguez-Gil A, et al. Population pharmacokinetic analysis of vancomycin in patients with hematological malignancies. Antimicrob Agents Chemother. 2005;49(12):4934-41.

19. Yasuhara M, Iga T, Zenda H, et al. Population pharmacokinetics of vancomycin in Japanese adult patients. Ther Drug Monit. 1998;20(02):139-48.

20. Zhong L, Minfang C, Ke C, et al. Population pharmacokinetics of vancomycin in chinese adult infection patients. Chin J Mod Appl Pharm. 2017;34(01):101-6.

21. Chang LIU, Shasha LIAO, Liqin ZHU, et al. Optimization of vancomycin and linezolid dosage regimen for treatment of Grampositive cocci infections. Tianjin Med J. 2016;44(8):1040-2.

22. Rong W, Huaijun Z, Yujie H, et al. Application of a population pharmacokinetic model for vancomycin in patients with severe neurosurgical disease. Chin J Infect Chemother. 2015;15(05):411-4.

23. Bing C, Wanhua Y, Weixia Z, et al. Pharmacokinetics of vancomycin in adult Chinese patients. Pharm Clin Res. 2013;21(06):605-8.

24. Xiao-rong H, Zhi-he L, Shuang-min J, et al. Population pharmacokinetics of vancomycin and prediction of pharmacodynamics in the Chinese people. Acta Pharm Sin. 2014;49(11):1528-35.

25. Fangjuan Weng. Population pharmacokinetic study of vancomycin. Strait Pharm J. 2013;25(07):207-9.

26. Wei W. Study on population pharmacokinetic and individualized administration of vancomycin. Fujian: Fujian Medical University; 2013.

27. Long M. Use of NONMEM method to establish population pharmacokinetic model of vancomycin in adults. In: The 4th therapeutic drug monitoring national annual conference of China. 2014; Abstract: 2.

28. Yang W, He B, Deng CH. Population pharmacokinetics of vancomycin from severe in patients with lower respiratory tract infection. Zhonghua Jie He He Hu Xi Za Zhi. 2017;40(03):205-9. 